\title{
The Relationship between Family Intimacy, Adaptability and Depression of Private College Students
}

\author{
Jia Yong, Yuan Chunlei \\ Foreign Trade and Business College, Chongqing Normal University, Chongqing, China \\ Email address: \\ cqriiayong@163.com (Jia Yong), 2467533090@qq.com (Yuan Chunlei) \\ To cite this article: \\ Jia Yong, Yuan Chunlei. The Relationship between Family Intimacy, Adaptability and Depression of Private College Students. American \\ Journal of Sports Science. Vol. 6, No. 3, 2018, pp. 83-87. doi: 10.11648/j.ajss.20180603.13
}

Received: April 12, 2018; Accepted: May 5, 2018; Published: May 28, 2018

\begin{abstract}
Aim: To study the relationship between family intimacy, adaptation and depression on private college students so as to provide theories to solve psychological problems of college students. Method: to apply stratified cluster sampling to 480 subjects from six private colleges in Chongqing, and to judge depression and family intimacy by CESD and FACESII-CV. Result: private college students' family intimacy and adaptability differ with sex; ideal adaptability differs with grade, while the other three show no difference among grades. Scores of depression are higher, and those scores show no distinction between sexes or among grades, probably because there's a large portion of depression. Conclusion: There is a negative correlation between family intimacy, adaptability and depression. Subjects with family intimacy and adaptability, especially ideal intimacy plays an important role in foretelling their depression. The aim is to provide the basis for psychological health guidance of college students.
\end{abstract}

Keywords: Family Intimacy, Family Adaptability, Depression, Private College

\section{Introduction}

With the development of society, mental health has become the important connotation of health. The private college students, as the subjects who are paid much attention by society, now face more severe dilemma than undergraduate students in job selection and employment. However, the fact is that we pay less attention on students from private college than on those who come from general universities, which leads to the result that effective counseling in mental health cannot approach private college students. And there are studies clearly showing that depression is a kind of common negative emotion among private college students. Depression is one of the reasons accounting for such phenomenon as suspension, withdrawal and suicide. Family intimacy can improve mental disease [2-4]. Foreign clinical studies have demonstrated that families that appear extreme behaviors in family intimacy and adaptability tend to face more negative phenomenon such as departure from home, mental, physical disease and children's inappropriate behavior, especially those family that lack of intimacy, family roles are disordered, or have unsteady regulations. The research of Cumsiell and Epstein shows that family intimacy and adaptability play a key role in the cause of juvenile delinquency. Experts among with Fang Xiaoyi wholly investigate the relation between family factors and the smoking behavior of middle school students, the study of which finds that family communication and intimacy have a significant influence on the juvenile smoking behavior. Yang Dehua, etc. has found that the poorer the degree of intimacy experienced by middle school students in the family, the more likely they would show depression and anxiety, which could lead to problematic behaviors. In the study of high school students, there is a significant correlation between rigid or loose families and variables such as anxiety, depression, helplessness and loneliness. It can be seen that the closeness of family members is significantly related to the individual mental health status $[1,2]$. Depression is both a low emotional state and a serious emotional disorder. Some scholars has pointed out that the incidence of depression in the general population appears a growing trend, which has become the main risk factors influencing human physical and mental health in the 21 st century. Depression often appears in the college student groups, and become one of the major factors that influence college students' study. Wang Jun and other researchers have pointed out that hostile tendencies, and emotional instability are introverted in personality and family environment factors that members do not actively participate in social and recreational activities, contradiction and girls, 
junior grade, rural background and poor grades are associated with depressive symptoms, among which the relationship between depressive symptoms and emotional instability is the closest $[2,5]$. This paper aims to explore the relationship between depression and the adaptability of family closeness and provide theoretical basis for the psychological health of college students.

\section{Methods}

\subsection{Test Object}

The research adopts the stratified cluster sampling method. Select 6 private colleges in Chongqing, among which freshman, sophomore, junior, and senior students are selected as the research objects, each grade 20 copies of questionnaires out of 480, recycling questionnaire 442, questionnaire recovery rate is $92.08 \%$, excluding invalid questionnaire to obtain effective questionnaire 357 , effective response rate is $80.77 \%$. There are 191 boys and 166 girls.

\subsection{Measuring Tool}

\subsubsection{Depression Symptom Scale}

The depression scale (CESD) was used in the flow adjustment center prepared by Radloff. It is applied to the general population of depressed mood to carry out the census. The evaluation is mainly about depressed mood and not depressive symptom group. The scale has 20 items that reflect the six aspects of depression: Depression, guilt and worthlessness, helplessness and hopelessness, mental mobility, loss of appetite, sleep disorders. Scale uses four-point scoring method, $1 \sim 4$, respectively, says "no", "occasional", "sometimes" or "continue", total score range is 0 to 60 . After negative questions recording, the higher score represents a more frequent depression symptom [6-8].

\subsubsection{Family Intimacy and Adaptability Scale}

Adopt the Chinese version of the second edition of the family intimacy and adaptability scale compiled by Olson etc. The Chinese version revised by Mr. Phillips, etc. revision, has good faith, validity, which is recognized as the self-assessment, including two sub-scales, and 30 projects totally. The scale mainly evaluates two aspects of family function, Intimacy: the relationship between family members; Adaptability: the ability changing according to the problems in the different family environment and different stages. The scale is assessed from "not" to "always" at 5. The scale requires the participants to answer two questions, one for the actual experience of their own family, and the other for the ideal family situation they hoped for.

\subsection{Data Processing}

SPSS 17.0 statistical software is used for data processing, data entry, sorting and statistical analysis.

\subsection{The Questionnaire Reliability}

Through the study on the reliability analysis of valid questionnaires, the cloning Bach coefficient of the depression scale (CESD) is $0.87,1 / 2$ coefficient is 0.858 ; Cloning Bach coefficient of family intimacy and adaptability is $0.946,1 / 2$ coefficient is 0.717 . In terms of the reliability analysis scores of two questionnaires, the scores are higher, which could clearly reflect the family closeness, adaptability and depression of college students in private colleges $[6,7]$.

\section{Results and Analysis}

\subsection{Statistical Analysis of Family Affinity and Adaptability of Private College Students}

Table 1 is the statistical table of familial intimacy and adaptability of college students. As can be seen in the table, the boys' actual intimacy, actual adaptability, intimacy, the ideal fitness scores are higher than girls', and the actual closeness, intimacy, the ideal adaptability in three dimensions presents significant gender difference, and the actual adaptability dimension shows significant difference in gender. From the point of each grade, grade 4 wins the highest score in the three dimensions including the actual intimacy, actual flexibility, ideal adaptability, grade 1 wins the highest scores in ideal intimacy, but only the ideal fitness shows very significant difference, the other three dimensions shows no significant differences in grade (Table 1).

Table 1. Statistical analysis of family closeness and adaptability of college students in private colleges.

\begin{tabular}{|c|c|c|c|c|c|}
\hline Dimension & Group & $\mathbf{N}$ & Mean & Std. & Sig. \\
\hline \multirow{2}{*}{ Actual intimacy } & male & 191 & 56.974 & 9.899 & \multirow{2}{*}{$0.003^{* *}$} \\
\hline & female & 166 & 53.697 & 10.630 & \\
\hline \multirow{2}{*}{ Actual adaptability } & male & 191 & 48.140 & 8.828 & \multirow{2}{*}{$0.027^{*}$} \\
\hline & female & 166 & 46.065 & 8.726 & \\
\hline \multirow{2}{*}{ Ideal intimacy } & male & 191 & 64.392 & 9.721 & \multirow{2}{*}{$0.000^{* *}$} \\
\hline & female & 166 & 59.863 & 10.904 & \\
\hline Ideal adaptability & male & 191 & 56.194 & 8.469 & $0.000^{* *}$ \\
\hline \multirow{4}{*}{ Actual intimacy } & 1 & 88 & 56.299 & 12.114 & \multirow{4}{*}{0.548} \\
\hline & 2 & 113 & 54.820 & 9.468 & \\
\hline & 3 & 117 & 54.921 & 9.707 & \\
\hline & 4 & 39 & 56.946 & 10.573 & \\
\hline \multirow{3}{*}{ Actual adaptability } & 1 & 88 & 47.860 & 10.192 & \multirow{3}{*}{0.200} \\
\hline & 2 & 113 & 46.280 & 7.920 & \\
\hline & 3 & 117 & 46.753 & 8.809 & \\
\hline
\end{tabular}




\begin{tabular}{llllll}
\hline Dimension & Group & N & Mean & Std. & Sig. \\
\hline & 4 & 39 & 49.492 & 7.829 & \\
Ideal intimacy & 1 & 88 & 63.069 & 11.450 & \\
& 2 & 113 & 60.941 & 8.101 & 0.432 \\
& 3 & 117 & 62.890 & 10.876 & \\
Ideal adaptability & 4 & 89 & 54.644 & 10.128 & \\
& 1 & 113 & 52.201 & 9.938 & $0.007^{* *}$ \\
& 2 & 117 & 56.183 & 8.222 & \\
\hline
\end{tabular}

Note: $* *$ means $\mathrm{p}<0.01, *$ means $\mathrm{p}<0.05$

\subsection{Statistical Analysis of Depression of Private College Students}

Table 2 is the statistics of depression scores of private college students, as you can see from the table, generally, the average score of depression is 16.891 , the standard deviation is 9.357 , and the author initially recommends 16 points as the possible cut-off point for depression. However, from the perspective of the overall average score of investigation, it has exceeded 16 points where there may exist depression problems (Table 2).

Table 2. Statistics of depression scores of private college students.

\begin{tabular}{llllll}
\hline \multicolumn{2}{l}{ Classification } & N & Mean & Std. & Sig. \\
\hline \multirow{3}{*}{ Gender } & male & 191 & 16.638 & 9.699 & \\
& female & 166 & 17.181 & 8.968 & 0.585 \\
& Total & 357 & 16.891 & 9.357 & \\
& 1 & 88 & 17.380 & 9.887 & \\
Grade & 2 & 113 & 17.044 & 8.712 & \multirow{2}{*}{0.814} \\
& 3 & 117 & 16.783 & 9.081 & \\
Total & 4 & 39 & 15.667 & 10.902 & \\
\hline
\end{tabular}

In terms of gender, the average score of male students is slightly lower than that of female students, but shows no significant difference. Grade 1 scores the highest, while grade 4 is the lowest. What's more, as the grade increases, depression scores appear a decreasing trend and depression scores among grades don't show significant differences.

Table 3 is the determination of depression scores of college students in private colleges. The original author initially recommends 16 points as the cut-off point for possible depression, corresponding to the 80 percentile. It is later suggested that 28 points should be used as a cut-off point for depression, corresponding to the 95 percentile. According to the score, the score is $0-15$ without depressive symptoms. 16-27 scores are judged to be depressive symptoms, and 28-60 are considered to be depressive symptoms. And then count the number in groups, there are 208 people without symptoms of depression, which accounts for $58.3 \%$ of the surveyed, 105 people may be at risk for depression symptoms, which accounts for $29.4 \%$ of the surveyed, 44 people have depressive symptoms, accounting for $12.3 \%$ of the surveyed people (Table 3). As can be seen from the survey, there may be a large proportion of people with depression and depressive symptoms $[7,8]$.

Table 3. Statistics of depression scores of private college students.

\begin{tabular}{lll}
\hline Scale & Number & Percentage\% \\
\hline Non-depressive symptoms & 208 & 58.3 \\
Potential depressive symptoms & 105 & 29.4 \\
depressive symptoms & 44 & 12.3 \\
Total & 357 & 100.0 \\
\hline
\end{tabular}

\subsection{Correlation Analysis of Family Closeness and Adaptability and Depression Among College Students in Private Colleges}

Table 4 is the correlation analysis of family intimacy and adaptability and depression of college students in private colleges. As is shown in the table, generally, depression and the actual intimacy, actual adaptability, ideal intimacy and adaptability of four dimensions present a very significant negative correlation.

Table 4. Correlation analysis of family intimacy and adaptability and depression of college students in private colleges.

\begin{tabular}{|c|c|c|c|c|c|}
\hline & & Actual intimacy & Actual adaptability & Ideal intimacy & Ideal adaptability \\
\hline \multirow{6}{*}{ Depression } & Male & $-0.293^{* *}$ & $-0.235^{* *}$ & $-0.371^{* *}$ & $-0.268^{* *}$ \\
\hline & Female & $-0.253^{* *}$ & $-0.185^{*}$ & $-0.378^{* *}$ & $-0.355^{* *}$ \\
\hline & Grade one & $-0.267^{*}$ & $-0.218^{*}$ & $-0.442^{* *}$ & $-0.395^{* *}$ \\
\hline & Grade three & -0.131 & -0.161 & $-0.238^{* *}$ & $-0.225^{*}$ \\
\hline & Grade four & $-0.488^{* *}$ & $-0.359^{*}$ & $-0.483^{* *}$ & $-0.317^{*}$ \\
\hline & Total & $-0.275^{* *}$ & $-0.214^{* *}$ & $-0.370^{* *}$ & $-0.308^{* *}$ \\
\hline
\end{tabular}

Note: ** means $\mathrm{p}<0.01, *$ means $\mathrm{p}<0.05$

In terms of gender, the family closeness and adaptability of male and female students are negatively correlated with depression scores. In terms of grade, the actual closeness and actual adaptability of grade 1 are negatively correlated with depression, and the ideal closeness and ideal adaptability are significantly negative. The actual closeness, ideal intimacy and ideal adaptability of grade 2 are all significantly negatively correlated with depression, and the actual 
adaptability is negatively correlated with depression. In the third grade, there is no significant correlation between the actual physical adaptation and depression, and the ideal closeness and ideal adaptability are significantly negatively correlated with depression $[9,10]$. The actual intimacy, ideal closeness and depression of grade 4 are negatively correlated with depression, and the actual adaptability and ideal adaptability are significantly negatively correlated with depression (Table 4).

Table 5. Correlation between familial intimacy and adaptability and depression evaluation levels in private schools.

\begin{tabular}{|c|c|c|c|c|}
\hline & Actual intimacy & Actual adaptability & Ideal intimacy & Ideal adaptability \\
\hline Non-depressive symptoms & $-0.339^{* *}$ & $-0.269^{* *}$ & $-0.333^{* *}$ & $-0.300^{* *}$ \\
\hline Potential symptoms & -0.097 & 0.042 & -0.039 & -0.014 \\
\hline Depressive symptoms & 0.227 & 0.111 & 0.004 & 0.105 \\
\hline
\end{tabular}

Table 5 is the correlation between familial intimacy and adaptability and depression evaluation levels in private schools, as can be seen from the table, the dimensions of family intimacy and adaptability without depressive symptoms and depression level have significant correlation. Actual closeness, intimacy, the ideal adaptability and the possible depression level of depression shows a negative correlation, but shows no significant difference, the actual adaptability possible depression levels are positively correlated with depression, and shows no significant difference (Table 5). Family closeness and adaptability are positively correlated with depressive symptoms and shows no significant difference $[6,7]$.

\subsection{Family Intimacy and Adaptability and Depression Regression Analysis of Private College Students}

Overall, family closeness and adaptability are negatively correlated with depression scores. Therefore, in order to understand which dimensions of family closeness can predict depression, it is necessary to gradually return the depression of predictors by applying family closeness adaptability dimension $[9,10]$.

Table 6. The regression of familial intimacy and adaptation and depression of private college students.

\begin{tabular}{lllll}
\hline & B & Beta & t & Sig. \\
\hline Constant & 39.497 & & 12.441 & 0.000 \\
Actual intimacy & -0.082 & -0.091 & -1.041 & 0.299 \\
Actual adaptability & -0.001 & -0.001 & -0.013 & 0.990 \\
Ideal intimacy & -0.250 & -0.281 & -3.078 & $0.002^{* *}$ \\
Ideal adaptability & -0.045 & -0.045 & -0.533 & 0.595 \\
\hline
\end{tabular}

Table 6 is the regression of familial intimacy and adaptation and depression of private college students, as can be seen from the table, family intimacy and adaptability are effective for the forecast of depression (Table 6). As can be seen from the dimensions, ideal intimacy dimension has the remarkable effectiveness for the forecast of depression.

\section{Conclusion}

Family intimacy and adaptability of private college students present differences in gender, and the ideal adaptability dimension presents difference in grade, the other 3 different dimensions don't present a grade differences. The scores of depression scores are higher in private colleges, and the scores of depression show no difference in gender and grade. There may be a large proportion of people with depression and depressive symptoms, accounting for 41.7 percent of the total population. There is a significant negative correlation between the degree of familial intimacy and adaptability of college students. There is a significant correlation between the dimensions of familial intimacy and adaptability and the level of depressive symptoms [8-10]. Family closeness and adaptability are positively correlated with depressive symptoms and shows no significant difference. The family closeness and adaptability of private college students are effective in predicting depression, and it can be seen from the dimension that the ideal intimacy dimension has a significant effect on the prediction of depression.

\section{References}

[1] Cumsiell P E, Epstein N B. Family cohesion, family adaptability, social support, and adolescent depressive symptom. Journal of Family Psychology, 1994, 8: 202-214.

[2] Fang Xiaoyi, Zheng Yu, Lin Danhus. The relationship between family factors and smoking behavior in junior high school students. [J]. Journal of psychology, 2001, 33(3):244-250.

[3] Yang Dehua. The relationship between family closeness, adaptability and depression, anxiety in junior high school students. [J]. Health psychology, 2001, 9(6):417-419.

[4] Wang Jun, Zhang Hongbo, etc. The relationship between depressive symptoms, personality and family environment in Anhui province. [J]. School health in China, 2009, 30(1):32-33.

[5] He Xinzhan, Long Haiyan. Study on the relationship between family intimacy and adaptability and understanding of social support. [J]. Theory and practice of Chinese rehabilitation, 2006, 12(7):634-636.

[6] Guo Lihua, Guan Ruiyuan, etc. Study on the adaptability and emotional state of familial intimacy between depressed patients and their families. [J]. Chinese journal of health psychology, 2008, 16(13):308-311

[7] Yang Lei. Study on the relationship between family intimacy, adaptability and peer trust of migrant children in the family of migrant workers [D]. Rudong University, 2017.

[8] Wang Bingying. The relationship between family intimacy and adaptability, depression and eating attitude [D]. Harbin Normal University, 2015. 
[9] Zhou Wanqiao. Research on the correlation and cultivation strategies of high school students' legal awareness and human nature, family closeness and adaptability [D]. Tianjin Normal University, 2014.

\section{Biography}

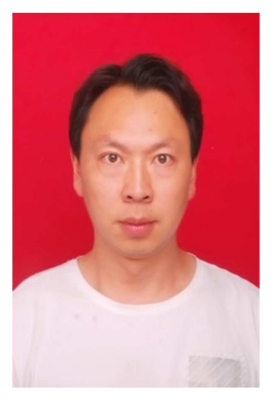

Jia Yong, male, born in 1979, Yun Yang, Chongqing, Master' degree, lecturer and director of Sport of Foreign trade and Business College of Chongqing Normal University, the author is mainly engaged in education management, and has presided over provincial and ministerial-level projects and 8 national sub-projects, edited three textbooks, published three core journals, participated in the Chongqing sports science paper report and has won many awards in the national competition on behalf of Chongqing.
[10] Zhu Yaxin. Study on the relationship between family intimacy, adaptability and relationship trust of college students [D]. Rudong University. 2014.

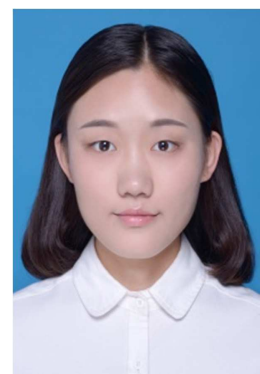

Yuan Chunlei, female, born in 1995, Xiang Yang, Hubei, Bachelor's degree, the author has published articles on American Scientific Research, and once been the interpreter of the Xin Jia Yuan Overseas Immigration Company in Chongqing; In November 2015, the author was appointed as the interpreter of Chinese-Italy industrial innovation Chongqing summit and has won the national scholarship and national motivational scholarships, advanced individual in science and technology innovation in Chongqing, the first prize of "FLTRP Cup" national college English writing contest and other Municipal, district awards. 